\title{
A study on Electron Oscillations in the Magnetosheath of Mars with Mars Express observations
}

\author{
Adriane M. de Souza ${ }^{1}$, Ezequiel Echer ${ }^{1}$, Mauricio J. A. Bolzam ${ }^{2}$ \\ and Markus Fränz ${ }^{3}$ \\ ${ }^{1}$ National Institute For Space Research \\ Sao Jose dos Campos, Brazil \\ ${ }^{2}$ Federal University of Goias, Jatai, Brazil \\ ${ }^{3}$ Max Planck Institute for Solar System Research, Göttingen, Germany, \\ email: adriane.souza@inpe.br
}

\begin{abstract}
Wavelet analysis was employed to identify the major frequencies of low-frequency waves present in the Martian magnetosheath. The Morlet wavelet transform was selected and applied to the electron density data, obtained from the Analyzer of Space Plasmas and Energetic Atoms experiment (ASPERA-3), onboard the Mars Express (MEX) spacecraft. We have selected magnetosheath crossings and analyzed electron density data. From a preliminary study of 502 magnetosheath crossings (observed during the year of 2005), we have found 1409 periods between 0.005 and $0.06 \mathrm{~Hz}$. The major frequencies observed were in the range $0.005-0.02 \mathrm{~Hz}$ with $58.5 \%$ of the 1409 frequencies identified.
\end{abstract}

Keywords. Mars Magnetosheath, Electron Oscillations, Wavelet Transform

\section{Introduction}

In planets lacking an internal magnetic field, the principal form of interaction between the solar wind and the body is through electro-magnetic induction. This induction can occur in conductive layers inside the planet or in an ionosphere, if the planet has an atmosphere. The induced electric currents flow through the planet or through the ionosphere and create forces that causes deceleration and deflection of the incident flow. Thereby, the deflected solar wind stream flows around a region similar to a magnetosphere created by an intrinsic magnetic field. Magnetospheres created due to this type of interaction are called induced magnetospheres (Cloutier \& Daniell (1973), Cloutier\& Daniell (1979), Luhman et al. (2004), Kivelson et al. (2007), Echer (2010) and Dieval et al. (2012)).

The magnetosheath of such unmagnetized planets has typically an addition of a new population of planetary ions to the hydrogen magnetoplasma, which drastically alters the dispersion of hydromagnetic waves and can produce new types of MHD (Magnetohydrodynamics) discontinuities in the transition region (Sauer et al. (1998)). The study of wave propagation is very important due to the fact that they have an important role in the energy and momentum transfer between the solar wind and the magnetosphere for example at Mars (Espley et al. (2004)). Consequently, these waves are related to the processes of atmospheric loss at Mars via interaction with the solar wind. Considering the importance to study waves in the Martian magnetosheath, the aim of this work is to determine the major frequency of electron oscillations in that region. 


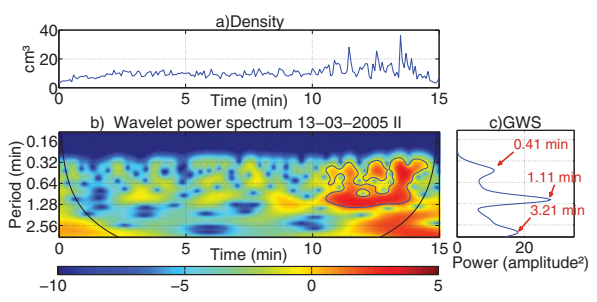

Figure 1. a- ASPERA $n_{e}$ time serie 13 March 2005. b- Spectrum Wavelet. c- GWS.

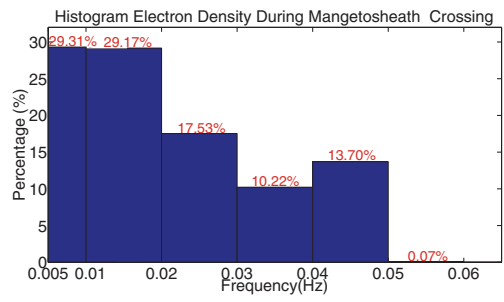

Figure 2. Histogram with the main frequencies identified in the GWS for all 2005 magnetosheath crossings.

\section{Data and methodology}

In order to perform this work, we have used electron density data $\left(n_{e}\right)$ from the electron spectrometer (ELS) within the Analyzer of Space Plasma and Energetic Atoms Experiment (ASPERA3) on board the spacecraft Mars Express (MEX) (Barabash et al. (2004)). A total of 502 magnetosheath crossings during the year 2005 have been analyzed.

The Wavelet Transform (WT) was applied on the data set studied in this work. The wavelet functions are generated by expansion, $\psi(t) \rightarrow \psi(2 t)$ and translations, $\psi(t) \rightarrow \psi(t+1)$ from a simple generating function, the wavelet-mother, given by $\psi_{a, b}(t)=$ $(1 / \sqrt{a}) \psi((t-b) / a)$. In this work we used the Morlet wavelet given by Torrence \& Compo (1998) in equation $\psi(t)=e^{i \zeta_{0} t} e^{-t^{2} / 2}$, where $\zeta$ is the dimensionless frequency. The WT applied on $\mathrm{f}(\mathrm{t})$ time is defined as $T W(a, b)=\int f(t) \psi_{a, b}(t)^{*} d t$, where $\psi_{a, b}(t)$ is the wavelet function and $\psi_{a, b}$ represents the complex conjugate thereof. The Global Wavelet Spectrum (GWS) was used to identify the most energetic periods in each magnetosheath crossing. The GWS is given by $G W S=\int|T W(a, b)|^{2} d b$.

\section{Results}

In order to obtain the main periods of low frequency electron oscillations in the Mars magnetoseath, the WT was applied to 502 magnetosheath crossings for data of $n_{e}$. Figure 1 shows an example of the WT results applied to the $n_{e}$ for the interval wherein the MEX crossed the Mars magnetosheath, between $10: 56$ UT and $11: 11$ UT on March 13, 2005. Figure 1-a presents $n_{e}$ time seires data and Figure 1-b the wavelet power spectrum. In the GWS of the Figure 1-c, we note the presence of three main periods: $0.41(0.04 \mathrm{~Hz})$, $1.11(0.02 \mathrm{~Hz})$ and 3.21 minutes $(0.005 \mathrm{~Hz})$, approximately.

After applying the WT to $n_{e}$ during the 502 magnetosheath crossings, 1409 frequencies were identified. These were divided into ranges to do a statistical analysis. Figure 2 shows the histogram with the results of the electron oscillation frequencies for these 502 magnetosheath crossings.

From the histogram we may note that the principal frequencies observed in the $n_{e}$ were in the range $0.005-0.01 \mathrm{~Hz}$ with $29.3 \%$ and in the range $0.01-0.02$ with $29.1 \%$ of the 1409 frequencies identified.Considering those two ranges, we have $58.5 \%$ of the frequencies in the interval of $0.005-0.02 \mathrm{~Hz}$.

These results agree with the findings by Winningham et al. (2006), where they used the integrated electron energy flux to study the electron oscillations in the induced magnetosphere of Mars. Those authors also observed a peak between 0.01 and $0.02 \mathrm{~Hz}$ in the magnetosheath. They have interpreted it as corresponding to the oxygen ion gyrofrequency in the Mars magnetosheath, calculated using data from the magnetometer from Mars Global Surveyor (Espley et al. (2004)). It is believed that for large scales 
(greater than the Debye Length $\left.\lambda_{D}\right)$, the plasma should be electrically neutral $\left(N_{e} \cong\right.$ $\left.N_{i+}\right)$. When ion wave modes, of large scale develop, they carry with them, the electrons which are associated with the oscillating ions. By this the electrons take their wavelike behavior. Electrons oscillate at higher frequency, but then also respond to lower frequencies to maintain quasi-equilibrium with positive ions. Therefore, when we observe in the ultra low frequency domain, the electrons trace the motion of the ions and the wave modes thereof (Winningham et al. (2006)). These authors also suggested that the bow shock may be the source of the oscillations.

\section{Summary}

In this paper we have determined the main frequencies of $n_{e}$ in the Martian magnetosheath. Given the obtained results it is emphasized that:

- The principal frequencies observed in $n_{e}$ were in the range $0.005-0.02 \mathrm{~Hz}$ with $58.5 \%$ of the 1409 frequencies identified;

- Those frequencies are near the local oxygen gyrofrequency in the Mars magnetosheath. Electrons trace the low frequency waves that affect ions;

- The bow shock may be the source of the oscillations;

As future work the authors intend to study all the Mars magnetosheath crossings during the whole MEX interval (2004-2015). We also will add electron temperature and pressure data and separate the analyzed data by position in the sheath, by solar cycle phases and solar wind conditions.

\section{Acknowledgments}

The authors would like to thank FAPESP for the financial support to AMS. The MJAB was supported by FAPEG (grant n. 201210267000905) and CNPq (grants n. $303103 / 2012-4)$. EE would like to thank to the Brazilian CNPq/PQ (302583/2015-7) agency for financial support.

\section{References}

Barabash et al. 2004, in: A. Wilson (ed.), Mars Express: the scientific payload. (Noordwijk, Netherlands: Esa publication), p. 121

Cloutier, P. A. \& Daniell, R. E. Jr. 1973, Planet. Space Sci., 21, 463

Cloutier, P. A. \& Daniell, R. E. Jr. 1979, Planet. Space Sci., 27, 1111

Dieval et al. 2012, Journal of Geophysical Research, 117, A06222

Echer, E. 2010, Revista Brasileira de Ensino de Fsica, 32, 230

Espley et al. 2004, Journal of Geophysical Research, 109,A4

Kivelson, M. G. \& Bagenal, F. 2007, in: L. A. MacFadden, P. R. Weissman, \& T. V. (eds.), Encyclpedia of the Solar System. (San Diego, CA: Academic), p. 519

Luhmann, J. G., Ledvina, S. A., \& Russel, C. T. 2004, Advances in Space Research, 33, 1905

Sauer, K., Dubinin, E., \& Baumgartel, K. 1998, Earth Planets Space, 50, 793

Torrence, C. \& Compo, G. P. 1998, Journal of Geophysical Research, 118, 1

Winningham et al. 2006, Icarus 182, 360. 